\title{
Cirugía de la rotura de músculo papilar por infarto miocárdico
}

\author{
Ricardo Zalaquett S, Fernando Cartajena de la Ma, \\ Pedro Becker R, Manuel Irarrázaval LI, Sergio Morán V. \\ Surgery of post myocardial infarction
papillary muscle rupture
}

Background: Papillary muscle rupture is a serious complication of myocardial infarction whose only treatment is surgery. Aim: To analyze our most recent surgical experience with papillary muscle rupture. Patients and Methods: The database of our Service was reviewed for the period 1995-2005, to identify patients with papillary muscle rupture. Then, the clinical records and operating protocols were analyzed. Survival and functional class of patients were assessed. Results: Twelve patients ( 7 men), aged 52 to 89 years, had papillary muscle rupture. They represented $2.2 \%$ of all mitral procedures for mitral insufficiency and $8.8 \%$ of mitral surgeries for ischemic mitral regurgitation, during the study period. In eight cases myocardial infarction was inferior, in three lateral and in one, anterior. In 10 patients, a mitral replacement was carried-out and in two the mitral valve was repaired. In seven patients, myocardial revascularization was performed. Mean lapse between infarction and surgery was 13.5 days ( 1 to 85 ). Two patients died in the perioperative period. Follow-up ranged from 1.7 to 120.4 months. Four patients died during follow-up, 2 due to a cardiovascular cause. Thus, at the end of follow-up 6 patients were alive. All were in functional class I-II. Conclusions: Papillary muscle rupture is an uncommon complication of myocardial infarction, with considerable operative and long-term mortality. However, survivors have good quality of life (Rev Méd Chile 2009; 137: 25-30).

(Key words: Heart rupture, post-infarction; Myocardial infarction; Papillary muscles)

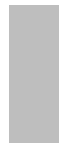

\footnotetext{
Recibido el 17 de junio, 2008. Aceptado el 12 de septiembre, 2008.

Departamento de Enfermedades Cardiovasculares, Facultad de Medicina, Pontificia Universidad Católica de Chile. Santiago de Chile.

anterno Facultad de Medicina, Pontificia Universidad Católica de Chile
}

$\mathrm{J}_{\mathrm{m}}^{\mathrm{m}} \mathrm{m}$ unto con la rotura de pared libre con tamponamiento cardiaco y la rotura del tabique interventricular con comunicación interventricular, la rotura de músculo papilar con insuficiencia mitral constituye una de las 3 complicaciones mecánicas agudas del infarto miocárdico ${ }^{1,2}$. Como para las

Correspondencia a: Dr. Ricardo Zalaquett S. Hospital Clínico, Pontificia Universidad Católica de Chile. Marcoleta 367, 6 piso. Teléfonos: 6333030-354 3231. Fax: 6390108.

E mail: rzalaque@med.puc.cl otras dos complicaciones, para la rotura de músculo papilar (RMP) la cirugía es la única alternativa de tratamiento realmente efectiva. El procedimiento quirúrgico consiste en el reemplazo protésico de la válvula mitral o en la reparación quirúrgica de ésta, con reimplante de la cabeza de músculo papilar desprendida y anuloplastia mitral complementaria con anillo protésico.

Si bien la RMP es una complicación muy conocida del infarto miocárdico, es poco frecuente, presentándose en alrededor de $1 \%$ de todas las 
muertes por infarto cardiaco ${ }^{1,2}$. Por lo mismo, las series quirúrgicas publicadas en la literatura son escasas e incluyen pocos pacientes, e incluso muchas de éstas se limitan a algunos casos clínicos.

Por nuestra parte, en 1995 comunicamos nuestra experiencia con el tratamiento quirúrgico de la insuficiencia mitral aguda por RMP en 9 pacientes, operados en el período comprendido entre 1980 y $1992^{3}$. En esta oportunidad analizamos nuestra experiencia y resultados con esta grave complicación mecánica del infarto miocárdico en los últimos 10 años, determinando su incidencia, métodos diagnósticos utilizados, forma de presentación clínica, procedimientos quirúrgicos realizados, mortalidad y morbilidad operatoria, sobrevida alejada y capacidad funcional de los sobrevivientes.

\section{PACIENTES Y MÉTODO}

El período de estudio se extendió entre enero de 1995 y diciembre de 2005, inclusive. Durante este período 6.732 pacientes fueron operados con circulación extracorpórea en nuestro Servicio de Cirugía Cardiovascular. Los pacientes con RMP fueron identificados a través de la base de datos electrónica de dicho servicio. Una vez identificados los pacientes, se procedió a la revisión de los protocolos operatorios y fichas clínicas, así como a la revisión de las ecocardiografías y coronariografías de éstos. Igualmente, se solicitó información al "Servicio de Registro Civil e Identificación de Chile" en cuanto a sobrevida y una vez identificado los sobrevivientes, éstos fueron contactados telefónicamente para responder una encuesta de evaluación clínica.

\section{RESULTADOS}

Entre enero 1995 y diciembre 2005 se identificaron en nuestra base de datos 12 pacientes operados por RMP, de los cuales 7 eran hombres. La edad promedio fue de 69 años con un margen de 52 a 89 años y una mediana de 66,5 años.

Incidencia quirúrgica. Estos 12 pacientes representaron $0,18 \%$ del total de las cirugías con circulación extracorpórea efectuadas en el período en estudio (6.732). Igualmente, durante este período se efectuaron un total de 542 cirugías por insuficiencia mitral, por lo que estos 12 pacientes representaron 2,2\% de éstas. Por otra parte, 137 de estos pacientes presentaban una etiología isquémica, por lo que la RMP representó 8,8\% de las operaciones por insuficiencia mitral isquémica (Tabla 1). El 91,2\% restante correspondió a insuficiencia mitral isquémica "ventricular" o "funcional", por dilatación del anillo mitral o tracción de los velos mitrales, por dilatación ventricular secundaria al infarto miocárdico.

Diagnóstico. El diagnóstico preoperatorio se confirmó por ecocardiografía en 3 casos, por angiografía con ventriculografía en 2 casos y en 7 casos por ambos procedimientos diagnósticos. En los 3 pacientes en que no se efectuó un estudio angiográfico con coronariografía la razón fue una grave inestabilidad hemodinámica.

Anatomía patológica. En 8 pacientes el infarto fue de pared inferior, en 3 pacientes de pared lateral y en 1 paciente de pared anterior, de acuerdo a criterios electrocardiográficos, ecocardiográficos o angiográficos.

Tabla 1. Representación porcentual de 12 pacientes operados por rotura de músculo papilar entre 1995 y 2005

\begin{tabular}{|lrr|}
\hline & No & \% RM P \\
\hline Total cirugías con CEC & 6.732 & 0,18 \\
Total cirugías por IM & 542 & 2,2 \\
Total cirugías por IM isquémica & 137 & 8,8 \\
\hline
\end{tabular}

RMP: rotura músculo papilar; CEC: circulación extracorpórea; IM: insuficiencia mitral 
Por otra parte, el músculo papilar roto correspondió en 7 casos al músculo papilar posterior y en los otros $5 \mathrm{al}$ anterior. Todos los pacientes presentaban regurgitación mitral masiva a la ecocardiografía o ventriculografía.

La Tabla 2 resume las características anatomopatológicas de estos 12 casos de RMP.

\section{Tabla 2. C aracterísticas anatomopatológicas de 12 pacientes operados por rotura de músculo papilar}

\begin{tabular}{|lrr|}
\hline & No & $\%$ \\
\hline Infarto & & \\
$\quad$ Inferior & 8 & 67 \\
Lateral & 3 & 25 \\
Anterior & 1 & 8 \\
Músculo papilar & & \\
$\quad$ Anterior & 5 & 42 \\
Posterior & 7 & 58 \\
\hline
\end{tabular}

Características clínicas. Diez pacientes presentaban edema pulmonar agudo, clínico y radiológico, al momento de la cirugía, 5 de los cuales requirieron apoyo con ventilación mecánica preoperatoria. Igualmente, 5 pacientes requirieron de drogas vasoactivas para su estabilización hemodinámica y 8 de un balón intraaórtico de contrapulsación. En 7 pacientes la cirugía debió efectuarse de urgencia, antes de las $12 \mathrm{~h}$ de hecho el diagnóstico, por deterioro hemodinámico progresivo. Los otros 3 pacientes con edema pulmonar agudo que no se operaron de urgencia fueron derivados desde otras instituciones.

Cirugía. El tiempo transcurrido entre el infarto miocárdico y la intervención quirúrgica fue en promedio de 13,5 días, con un margen entre $1 \mathrm{y}$ 85 días y una mediana de 1,5 días.

En 10 pacientes se efectuó un reemplazo valvular mitral, en 7 de éstos con prótesis mecánica y en 3 con prótesis biológica. A los 2 pacientes restantes se les efectuó una reparación valvular mitral con reimplante de la cabeza de músculo papilar desprendida al músculo papilar adyacente y anuloplastia con anillo protésico de CarpentierEdwards $^{\mathrm{TM}}$. En todos los casos, la válvula mitral fue expuesta a través de un acceso auricular izquierdo transeptal y superior combinado ${ }^{4}$.

En 7 pacientes se efectuó una revascularización miocárdica, con un promedio de 1,7 puentes por paciente.

Dos pacientes fallecieron en el período perioperatorio, por lo que la mortalidad operatoria fue de $17 \%$. En ambos se había efectuado un reemplazo valvular mitral, en 1 con una prótesis mecánica, el que falleció a los 7 días de la operación, y en otro con una prótesis biológica, el que falleció al segundo día de la cirugía. Estos 2 pacientes fueron operados de urgencia. La causa de muerte fue insuficiencia cardiaca en ambos. El paciente fallecido al séptimo día desarrolló, además, una falla multisistémica.

De los sobrevivientes a la cirugía, un paciente fue reintervenido por hemorragia postoperatoria y otro presentó una sepsis de origen respiratorio que requirió de ventilación mecánica prolongada con traqueostomía e insuficiencia renal aguda. Finalmente, un paciente evolucionó con una escara sacra y retardo de cicatrización de la safenectomía y otro presentó un hematoma inguinal.

Resultados alejados. El seguimiento se cerró en agosto de 2006 y se completó en 100\% de los casos, con un promedio de 65,8 meses y un margen de 1,5 a 120 meses. Durante el seguimiento fallecieron 4 pacientes. Dos de éstos lo hicieron por insuficiencia cardiaca; en uno de ellos se había efectuado un reemplazo mitral, el que falleció a los 1,5 meses de la operación, y en el otro una reparación valvular mitral, el que falleció 12,5 meses después de la reparación mitral. Los otros 2 pacientes fallecieron por causas no cardiacas. Así, al término del seguimiento 6 pacientes (50\%) se encontraban vivos. La capacidad funcional en todos los sobrevivientes fue I a II.

\section{DisCUSIÓN}

La insuficiencia mitral isquémica puede ser funcional, también llamada ventricular, porque el mecanismo de ésta es sólo la tracción, simétrica o asimétrica, de los músculos papilares por la dilatación ventricular, y, en menor medida, la dilatación del anillo mitral, o anatómica o valvular, por rotura de múscu- 
lo papilar ${ }^{1,5-8}$. Si bien la insuficiencia mitral isquémica ventricular es la situación más frecuente, la RMP es la forma de insuficiencia mitral isquémica en general más conocida por la mayoría de los médicos y cirujanos, a pesar de que en la práctica es muy infrecuente, representando en la mayoría de los casos, como en nuestras 2 series, reciente y antigua, una bajísima proporción de los procedimientos cardioquirúrgicos en general y de los procedimientos mitrales por insuficiencia en particular ${ }^{4,7-9}$. Específicamente, en nuestra experiencia, no alcanza a representar $10 \%$ de los procedimientos mitrales por insuficiencia mitral de causa isquémica. Esta discrepancia de apreciación se puede explicar por la gravedad clínica inmediata de la RMP, si bien en el mediano y largo plazo la insuficiencia mitral isquémica ventricular termina siendo de mayor mortalidad, o porque el mecanismo anatomopatológico de la regurgitación mitral es claramente entendible, a diferencia de la insuficiencia mitral ventricular.

Igualmente, resulta en cierta medida sorprendente esta baja incidencia de la RMP, puesto que los músculos papilares constituyen la porción del miocardio de mayor grosor de éste y al ser la irrigación del corazón de tipo terminal, por vasos perforantes que se originan en las arterias coronarias epicárdicas, los músculos papilares son, por lo tanto, la porción del miocardio más susceptible a la isquemia y al infarto miocárdico ${ }^{10-12}$.

De hecho, el músculo papilar posterior, que sólo recibe su irrigación de la arteria descendente posterior, sea esta rama de la arteria coronaria derecha o de la arteria circunfleja, lo que lo deja en situación de mayor labilidad en comparación al músculo papilar anterior, que es irrigado por ramos perforantes provenientes de ramas diagonales de la arteria descendente anterior y ramos marginales de la arteria circunfleja, es el que efectivamente con mayor frecuencia resulta comprometido, como lo ha sido en nuestra experiencia, previa y actual ${ }^{1,2,10-12}$.

La RMP se presenta en general precozmente en relación al infarto, en nuestra experiencia, más frecuentemente en los primeros 2 días de éste, si bien en algunos pacientes el diagnóstico y la intervención quirúrgica pueden ser muy tardíos, como en esta serie reciente, hasta 85 días después del infarto ${ }^{1-3}$.

Por otra parte, la RMP es una complicación gravísima del infarto miocárdico. Cuando es todo el músculo papilar el que se desprende de la pared ventricular, la muerte es en general inmediata, por bajo gasto cardiaco agudo y edema pulmonar masivo ${ }^{1-3,12}$. Los pacientes que sobreviven a una RMP y que logran recibir tratamiento quirúrgico son, en general, aquellos en los que la rotura compromete una porción o cabeza de músculo papilar. Pero, aunque sobreviven a la rotura aguda, son pacientes muy graves. Tanto en nuestra experiencia previa como reciente, y en casi todas las pequeñas series publicadas, la mayoría de los pacientes requieren de ventilación mecánica, balón intraaórtico de contrapulsación y drogas vasoactivas ${ }^{1-3,13}$. Sin embargo, a diferencia de la insuficiencia cardiaca aguda por pérdida de masa muscular por un infarto extenso, la que en general se acompaña también de insuficiencia mitral funcional, esta insuficiencia cardiaca es potencialmente reversible y el pronóstico alejado mejor ${ }^{1,2,5,6}$.

El balón intraaórtico de contrapulsación es el procedimiento terapéutico más efectivo para estabilizar al paciente previo a la cirugía, ya que al aumentar la perfusión coronaria, a la vez que disminuir el trabajo cardiaco y el gradiente de presión entre la aurícula izquierda y el ventrículo izquierdo, disminuye simultáneamente la isquemia miocárdica y la regurgitación mitral ${ }^{1,14}$. En nuestra experiencia es utilizado en la mayoría de los pacientes, incluso antes que las drogas vasoactivas.

El diagnóstico definitivo de RMP lo da en la actualidad la ecocardiografía, la que no sólo confirma la existencia de una insuficiencia mitral masiva, sino que, además, demuestra la cabeza de músculo papilar desprendida moviéndose erráticamente entre la aurícula y el ventrículo izquierdo, en una típica imagen en "boleadora" (Figura 1) ${ }^{15,16}$. Sin embargo, la angiografía es fundamental para evaluar la magnitud de la enfermedad coronaria, la que si bien en general es de un solo vaso, también puede serlo de múltiples ${ }^{1,2}$. De hecho, en nuestras 2 series, más de la mitad de los pacientes requirió de revascularización miocárdica y la mayoría de ellos con más de un puente coronario.

Si bien en lo que a la válvula mitral insuficiente respecta siempre la reparación será el mejor procedimiento terapéutico, en la RMP ésta no se efectúa en la mayoría de los pacientes, como en nuestras 2 series, reciente y tardía ${ }^{1-5,7,9,13,17-20}$. 


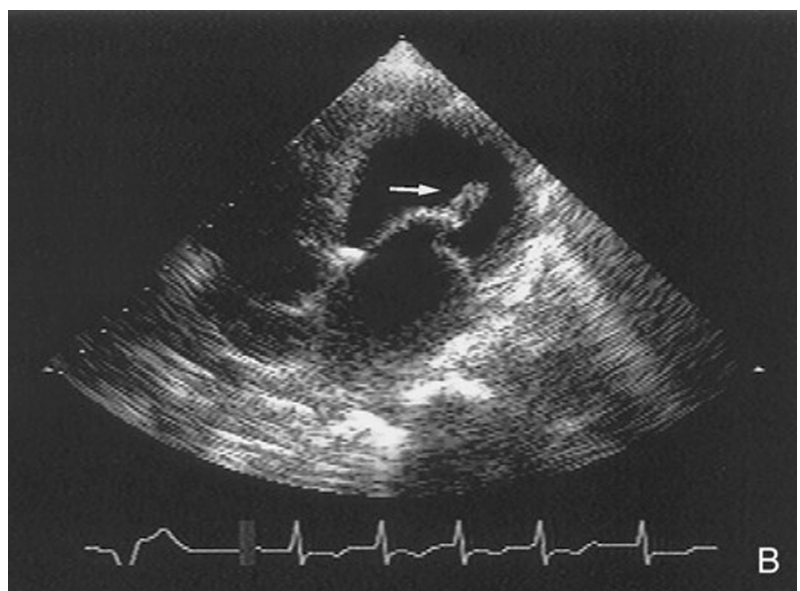

Figura 1. Imagen ecocardiográfica de cabeza de músculo papilar desprendida (flecha) desplazándose erráticamente entre aurícula y ventrículo izquierdo, en típica imagen en "boleadora".

Probablemente esto está determinado por el entrenamiento que tengan los cirujanos en reparación mitral y su disposición a efectuar un procedimiento que puede estar expuesto a un fracaso y requerir de un nuevo período de circulación extracorpórea, en pacientes operados de urgencia, con frecuencia de noche, así como por la dificultad de exponer la válvula mitral cuando la aurícula izquierda es de tamaño normal, como es en la RMP, pero, fundamentalmente, por el temor a reimplantar una cabeza de músculo papilar desprendida en un tejido miocárdico que está evolucionando un infarto.

En cualquier caso, una adecuada exposición de la válvula mitral es fundamental, por lo que dado que en la RMP la aurícula izquierda es de tamaño normal, es decir, con un diámetro mayor de alrededor de $4 \mathrm{~cm}$, hemos utilizado en todos los casos un acceso auricular izquierdo transeptal y superior combinado, el que disminuye la distancia a la válvula mitral y facilita la exposición de ésta y su aparato subvalvular, al no tener el tabique interauricular y la aurícula derecha encima, como sucede en el acceso auricular izquierdo clásico, por detrás y paralelo al surco interauricular ${ }^{4}$.

La mortalidad operatoria de la RMP es elevada, en la mayoría de las series publicadas, como en nuestras 2 experiencias, actual y previa, de alrededor de $20 \%$. Sin embargo, los resultados alejados son más satisfactorios en cuanto al estado sintomático de los pacientes, aunque también hay una mortalidad tardía de consideración, en parte explicada por la edad avanzada de los pacientes, lo que los hace susceptibles a distintas causas de muerte, no sólo cardiovascular1-3,7,13,14. En esta experiencia reciente, la mitad de los pacientes estaban vivos al término del seguimiento, incluyendo la mortalidad operatoria. Pero, más importantemente, todos ellos estaban en capacidad funcional I-II.

\section{CONCLUSIONES}

En esta experiencia, como en nuestra experiencia previa, la RMP es una complicación poco frecuente, pero muy grave del infarto miocárdico, para la cual la única alternativa de tratamiento efectiva es la operación, la que en la mayoría de los casos, por distintas razones, consiste en un reemplazo protésico de la válvula mitral. La mortalidad operatoria es considerable, de alrededor de $20 \%$, y si bien la sobrevida alejada, incluyendo ésta, es limitada, los sobrevivientes a la cirugía gozan de una buena capacidad funcional. 


\section{REFERENCIAS}

1. Kouchoukos NT, Biackstone EH, Doty DB, Hanley FL, KaRP RB. Cardiac surgery. $3^{\text {rd }}$ Ed. Philadelphia: Elsevier Science (USA), 2003; 472-9.

2. Zalaquett R. Cirugía de las complicaciones mecánicas agudas del infarto miocárdico. Rev Chil Cirugía 1995; 47: 2-7.

3. Zalaquett $R$, Morán S, Irarrázaval $M$, Becker P, Maturana G, Navarro M et al. [Papillary muscle rupture in acute myocardiacdial infarction]. Rev Méd Chile 1995; 123: 199-206.

4. Zalaquett R, Morán S, Irarrázaval M, Maturana G, NavarRo M, Braun S et al. Acceso auricular izquierdo transeptal y superior combinado. Rev Chil Cirugía 1993; 45: 225-8.

5. LuNg B. Management of ischaemic mitral regurgitation. Heart 2003; 89: 459-646.

6. LeVINe RA, Schwammenthal E. Ischemic mitral regurgitation on the threshold of a solution. From paradoxes to unifying concepts. Circulation 2005; 112: 745-58.

7. Zalaquett $R$, Garrido L, Morán S, Irarrázaval $M$, Becker P, Maturana G et al. [Surgery for ischemic mitral regurgitation]. Rev Méd Chile 2002; 130: 9-16.

8. Ourry JH, Ciaveland JC, Durán GC, Angel WW. Ischemic mitral valve disease: Clasification and sistemic approach to management. J Card Surg 1994; 9 (2 suppl): 262-73.

9. Guimnov AM, Wierup PN, Biackstone EH, Bishay ES, Cosgrove DM, White J ET AL. Is repair preferable to replacement for ischemic mitral regurgitation? J Thorac Cardiovasc Surg 2001; 122: 1125-41.

10. Perloff JK, Roberts WC. The mitral apparatus: Functional anatomy of mitral regurgitation. Circulation 1972; 46: 227-39.
11. RoBeRTs WC. Morphologic features of the normal and abnormal mitral valve. Am J Cardiol 1983; 51: 1005-28.

12. RoBeRTS WC, COHEN LS. Left ventricular papillary muscles. Description of the normal and a survey of conditions causing them to be abnormal. Circulation 1972; 46: 138-54.

13. Kilien DA, Reed WA, Wathanacharoen S, Beauchamp G, RUTHERFORD B. Surgical treatment of papillary muscle rupture. Ann Thorac Surg 1983; 35: 243-8.

14. Gold HL, LeINBACh RC, SANDERS CA. Intraaortic balloon pumping for ventricular septal defect or mitral regurgitation complicating acute myocardial infarction. Circulation 1973; 47: 1191-6.

15. Erbel R, Schweizer P, Bardos P, Meyer J. Twodimensional echocardiographic diagnosis of papillary muscle rupture. Chest 1981; 79: 595-602.

16. Nishimura RA, Schaff HV, Shub C, Gersh BJ, Edwards WE, TајIK AJ. Papillary muscle rupture complicating acute myocardial infarction: Analysis of 17 patients. Am J Cardiol 1983; 51: 373-81.

17. Zalaquett R, Chamorro G, Braun S, Garrido L, Howard M, Morán S ET aL. [Long-term results of reconstructive surgery for mitral insufficiency]. Rev Méd Chile 1999; 127: 1093-100.

18. Rankin JS, Feneley MP, Hickey M, Muhlbaler LH, WECHLER AS, FLOYd RD ET AL. A clinical comparison of mitral valve repair versus valve replacement in ischemic mitral regurgitation. J Thorac Cardiovasc Surg 1988; 95: 165-77.

19. Hendren WG, Nemec JJ, Lytle BW, Loop FD, Taylor PC, Stewardt RW ET AL. Mitral valve repair for ischemic mitral insufficiency. Ann Thorac Surg 1991; 52: 1246-52.

20. Al-Radi OO, Austin PC, Tu JV, David TE, Yau TM. Mitral repair versus replacement for ischemic mitral regurgitation. Ann Thorac Surg 2005; 79: 1260-7. 